\title{
New Planar High Q Active Resonator and Its Application to Low Phase Noise Oscillators
}

\author{
Young-Taek Lee, Jaechun Lee, and Sangwook Nam \\ School of Electrical Engineering \& Computer Science, Seoul National University, Seoul, Korea
}

\begin{abstract}
This paper presents a new technique to design a high $Q$ active resonator using an amplifier which is located at the loop of the resonator. By adjusting the phase of the loop, the equivalent negative resistance is generated at the one coupling port of the resonator, which leads to high $Q$ property without the negative resistance circuit. The bandstop resonator using this technique shows a high $Q$ factor of 548.62 from measurement at $10 \mathrm{GHz}$ resonant frequency. To demonstrate the validity of the proposed active resonator, an oscillator incorporated with this active resonator was designed, fabricated and measured. The phase noise performance of the active resonator oscillator is $\mathbf{- 1 1 2 . 6}$ $\mathrm{dBc} / \mathrm{Hz}$ at $100 \mathrm{KHz}$ offset, which is reduced by $11.3-14.4 \mathrm{~dB}$ compared to the passive resonator oscillator.
\end{abstract}

Index Terms - Active resonator, quality factor, oscillator, phase noise.

\section{INTRODUCTION}

The modern communication systems require the high $\mathrm{Q}$ resonators for bandpass filters, bandstop filters, duplexers and oscillators. The dielectric resonator and cavity resonator are promising elements for these applications but they have three dimensional structure and their sizes are bulky. Therefore they limit the on chip IC realization and are not adequate for mass production.

Especially, the high Q resonators are essential for low phase noise oscillators. The phase noise is the most important parameter because it determines the overall performances of the communication systems. In recent years, there have been many attempts to reduce the phase noise of the planar oscillators which have some advantages for low cost and reliability [1]-[6]. Nevertheless their phase noise characteristics are inferior due to their poor Q factors of the planar resonators.

To overcome the limitation of the $\mathrm{Q}$ factors of the planar resonators, various researches to design the active high Q resonators have been reported and they are good candidates for high performance filters and oscillators. Usually the active high $\mathrm{Q}$ resonators are implemented based on the negative resistance circuit, which is used to compensate the loss of the resonator and as a result the high Q property can be obtained [7]-[12].

Although the negative resistance circuits are widely used to implement the high $\mathrm{Q}$ active resonators, they have some following drawbacks. First, the schematic is somewhat complicated because they must have the feedback element and the matching circuits to produce the negative resistance. Second, spurious oscillation can be occurred if oscillation start-up condition is satisfied. Hence careful design must be provided to generate the negative resistance and to prevent the unwanted oscillation.

In this paper, a new method to design a high $\mathrm{Q}$ active bandstop resonator without negative resistance circuit and its application to low phase noise oscillators are proposed. Instead of using the negative resistance circuit, an amplifier is located between two coupling ports of the resonator. To validate the applicability of this active resonator to low phase noise oscillators, a series feedback oscillator with the active resonator was designed and measured.

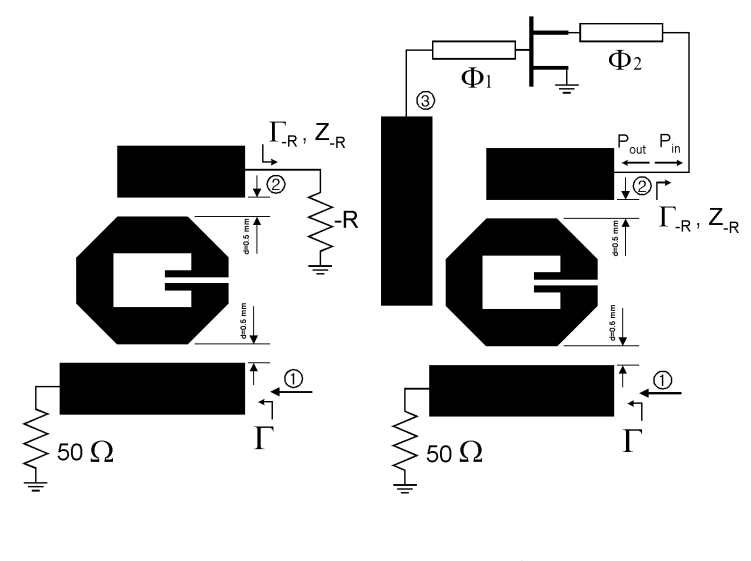

(a)

(b)

Fig. 1. The high $\mathrm{Q}$ active bandstop resonator using (a) the negative resistance circuit. (b) the amplifier.

\section{OPERATION PRINCIPLE OF High Q ACTIVE RESONATOR}

Fig. 1 (a) shows one possible method to design a high $\mathrm{Q}$ active bandstop resonator using the negative resistance circuit. To realize the negative resistance circuit, an active 
device with feedback element is needed, which causes the complex structure and oscillation problem as mentioned above.

The schematic of the proposed high Q active bandstop resonator using the amplifier is shown in Fig. 1 (b). Unlike the previous one, additional coupling port (port 3) is placed and an amplifier is located between the port 2 and port 3.

To understand the operation principle of the proposed active resonator, the analysis of the negative resistance circuit in Fig. 1 (a) should be performed. Basically the negative resistance plays a role of reflection type amplification of signal, which means that the reflection coefficient $(\Gamma-\mathrm{R})$ seen from the port 2 to the negative resistance circuit exceeds one near the resonant frequency. The active resonator proposed in this paper shows similar operation. A signal is injected to the port 1 and coupled to the port 2 and port 3 . Then the signal from the port 3 is amplified and injected to the port 2 in opposite direction as shown in Fig. 1 (b). By adjusting the phases $\left(\Phi_{1}, \Phi_{2}\right)$ of the transmission lines located at the two coupling ports, the $\Gamma-\mathrm{R}\left(\mathrm{P}_{\text {out }} / \mathrm{P}_{\text {in }}\right)$ is larger than one, which suggests that the equivalent negative resistance is generated at the port 2. From the circuit simulation, it is calculated that the $\Gamma-\mathrm{R}$ is $5 \mathrm{~dB}$ and $\mathrm{Z}-\mathrm{R}$ is $-13.9+4.3 \mathrm{j}$ ohm at the resonant frequency as shown in Fig. 2. In conclusion, the high $Q$ active resonator can be designed simply using the amplifier without the complicated negative resistance circuit.

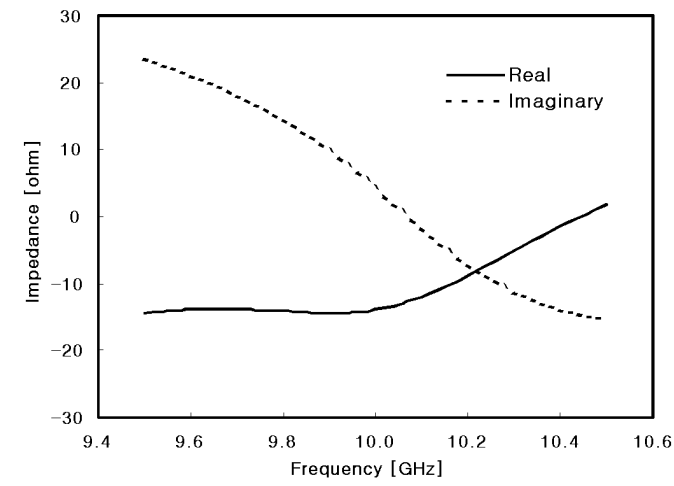

Fig. 2. The calculated input impedance $\left(Z_{-R}\right)$ seen from the port 2 in Fig. 1 (b).

One may doubt that there is possibility of oscillation because the amplifier is located in the closed loop. However the coupling coefficient between port 2 and port 3 is very weak (below $-10 \mathrm{~dB}$ ), which indicates that the loop gain of the closed loop is less than unity at whole frequency bands as shown in Fig. 3. Therefore no oscillation can be occurred.

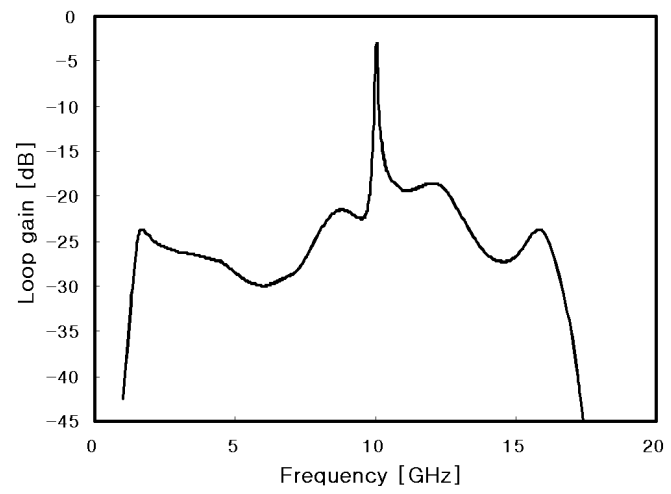

Fig. 3. The characteristic of the loop gain. The port 1 is terminated to $50 \Omega$.

The active resonator was fabricated using the Teflon substrate of $0.504 \mathrm{~mm}$ thickness and dielectric constant of 2.52. The TC 2381 medium power device was used as an amplifier.

The simulated and measured results of the proposed active bandstop resonator are in good agreement as shown in Fig. 4. The loaded quality factor from the measurement is calculated to be 548.62. The measured result of the passive hairpin resonator strongly coupled to the microstrip line is also shown in Fig. 4 and the loaded quality factor is calculated to be 65.72 .

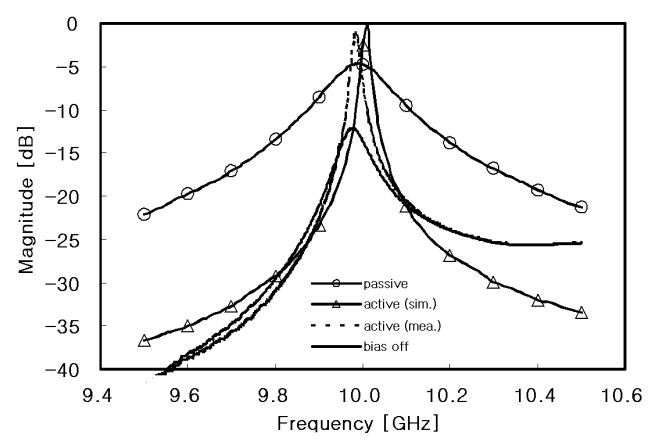

Fig. 4. Frequency responses of the passive resonator (measurement), the active resonator (simulation and measurement), and the active resonator when the bias is off (measurement). 


\section{Design of Oscillator Using High Q Active RESONATOR}

The schematic of the active resonator oscillator is depicted in Fig. 5. The active device for oscillator is NE 32584 HEMT device and it was designed by using the nonlinear simulation approach [13]. Its oscillation frequency is $10 \mathrm{GHz}$, which is the resonant frequency of the active resonator. The source and drain circuit of the passive resonator oscillator is identical to those of the active resonator oscillator and the gate circuit of that was replaced by the passive resonator.

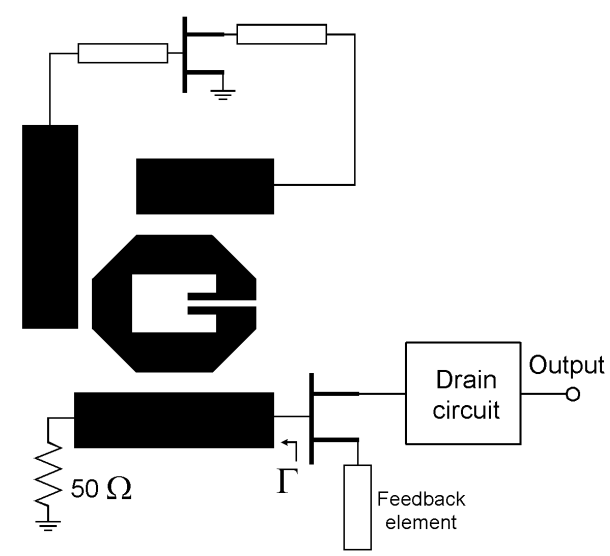

Fig. 5. The schematic of the active resonator oscillator.

\section{Measured Results of THE ACtive Resonator OSCILLATOR}

The phase noise characteristics of the active and passive resonator oscillators were measured using HP $8566 \mathrm{~B}$ spectrum analyzer. Fig. 6 depicts the measured phase noise results of these two oscillators depending on the bias voltages. The phase noise of the active resonator oscillator is lower than that of the passive resonator oscillator by 11.7-14.4dB.

The pulling figures of these two oscillators were measured to compare the external quality factors of them [14]. The external Qs of the active resonator oscillator and passive resonator oscillator are 1211.2 and 216.6 respectively, which indicates that the ratio of the external Qs is 5.59 and $14.9 \mathrm{~dB}$ phase noise improvement is expected. This is in agreement as the measured phase noise results.

The ratio of the external Qs of the oscillators is slightly different to that of the active and passive resonators. It is believed that this is because the operating point of the device of the active resonator in oscillator is changed from that of the device of the active resonator itself.

However the quality factor would be more increased and the phase noise be reduced through further optimization of the active resonator.

The output spectrum of the active resonator oscillator is illustrated in Fig. 7 and it shows $-112.6 \mathrm{dBc} / \mathrm{Hz}$ at $100 \mathrm{KHz}$ offset. It is comparable to the phase noise performance in [15], which used a low noise silicon device for VCO.

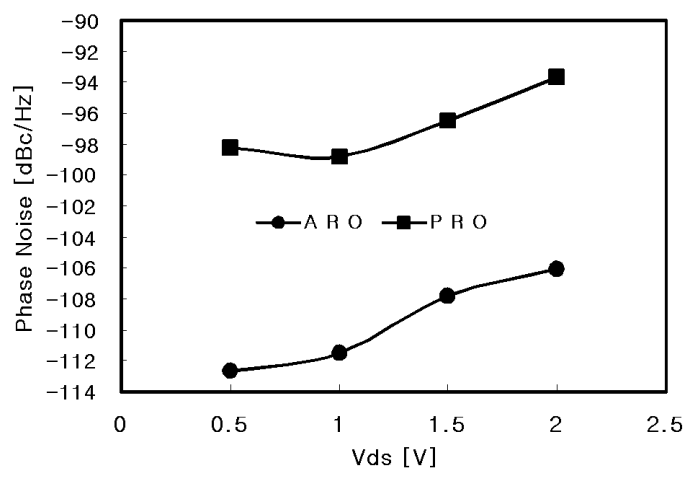

(a)

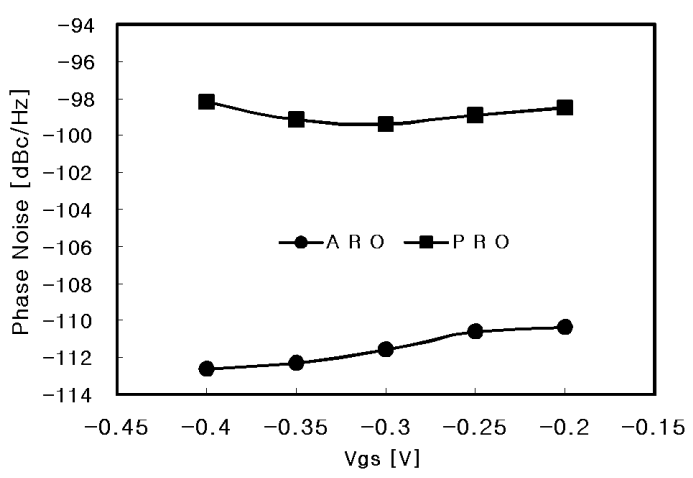

(b)

Fig. 6. The measured phase noise characteristics of the active resonator oscillator (ARO) and the passive resonator oscillator (PRO) at $100 \mathrm{KHz}$ offset. (a) depending the drain bias voltages $(\mathrm{Vgs}=-0.4 \mathrm{~V})$. (b) depending on the gate bias voltages $(\mathrm{Vds}=0.5 \mathrm{~V})$.

\section{CONCLUSION}

The new high $\mathrm{Q}$ active bandstop resonator using the amplifier for planar structure has been presented. It overcomes the difficulties of the active high $\mathrm{Q}$ resonator using the negative resistance circuit such as complexity 
and oscillation problems. Moreover its design procedure is very simple. The fabricated active resonator shows a high loaded Q of 548.62. A $10 \mathrm{GHz}$ series feedback oscillator utilizing this new active resonator was designed and measured to verify the possibility of application. The phase noise of the active resonator oscillator was measured and compared to that of the passive resonator oscillator. The active resonator oscillator shows low phase noise performance of $-112.6 \mathrm{dBc} / \mathrm{Hz}$ at $100 \mathrm{KHz}$ offset, which is reduced by $11.3-14.4 \mathrm{~dB}$ compared to the passive resonator oscillator.

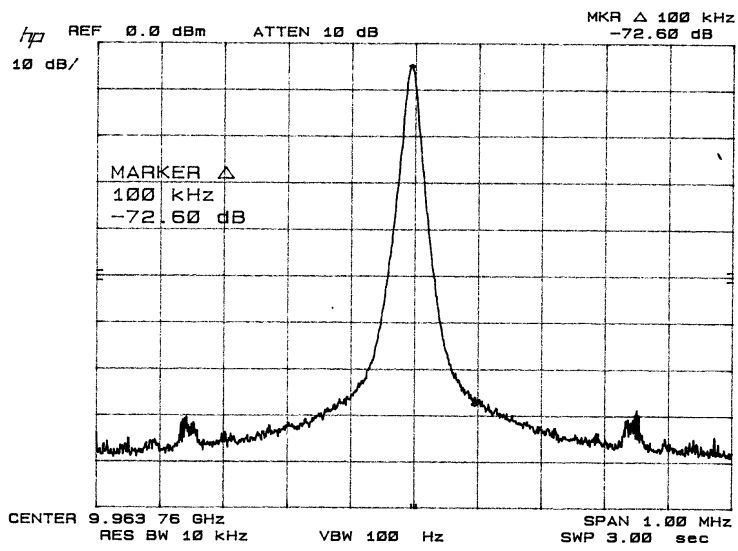

Fig. 7. The output spectrum of the active resonator oscillator $(\mathrm{Vgs}=-0.4 \mathrm{~V}, \mathrm{Vds}=0.5 \mathrm{~V})$

\section{ACKNOWLEDGEMENT}

This work was supported by Brain Korea 21 project.

\section{REFERENCES}

[1] Y.-T. Lee, J.-S. Lim, J.-S. Park, D. Ahn, and S. Nam, “A novel phase noise reduction technique in oscillators using defected ground structure," IEEE Microwave and Wireless Components Lett., vol. 12, no. 2, pp. 39-41, Feb. 2002.

[2] Y.-T. Lee, J.-S. Lim, C.-S. Kim, D. Ahn, and S. Nam, "A compact-size microstrip spiral resonator and its application to microwave oscillator," IEEE Microwave and Wireless Components Lett., vol. 12, no. 10, pp. 375-377, Oct. 2002.

[3] L. Dussopt, D. Guillois, and G. M. Rebeiz, "A low phase noise silicon $9 \mathrm{GHz} \mathrm{VCO}$ and $18 \mathrm{GHz}$ push-push oscillator," 2002 IEEE MTT-S Int. Microwave Symp. Dig., vol. 2, pp. 695-698, June 2002.

[4] W.-C. Lee, S.-C. Lin, and C.-K. C. Tzuang, "Planar realization of low phase noise $15 / 30 \mathrm{GHz}$ oscillator/doubler using surface mount transistors," IEEE Microwave and Wireless Components Lett., vol. 13, no. 1, pp. 10-12, Jan. 2003.

[5] Y. Cassivi and $\mathrm{K}$. Wu, "Low cost microwave oscillator using substrate integrated wavegiude cavity," IEEE Microwave and Wireless Components Lett., vol. 13, no. 2, pp. 48-50, Feb. 2003.

[6] L.-H. Hsieh and K. Chang, "High-efficiency piezoelectrictransducer-tuned feedback microstrip ring-resonator oscillators operating at high resonant frequencies," IEEE Trans. Microwave Theory \& Tech., vol. 51, no. 4, pp. 11411145, Apr. 2003

[7] C.-Y. Chang and T. Itoh, "Microwave active filters based on coupled negative resistance method," IEEE Trans. Microwave Theory \& Tech., vol. 38, no. 12, pp. 1879-1884, Dec. 1990

[8] Y. Yamamoto, K. Kawasaki, and T. Itoh, "A MESFETcontrolled X-band active bandpass filter," IEEE Microwave and Guided Wave Lett., vol. 1, no. 5, pp. 110-111, May 1991.

[9] J. Lin and T. Itoh, "Tunable active filters using threeterminal MESFET varactor," 1992 IEEE MTT-S Int Microwave Symp. Dig., vol. 2, pp. 921-924, May 1992.

[10] Y.-H. Cho, S.-C. Hong, Y. -S. Kwon, "A low-power monolithic GaAs FET bandpass filter based on negative resistance technique," IEEE Microwave and Guided Wave Lett., vol. 8, no. 4, pp. 161-163, April 1998.

[11] Y. Yamamoto, Y. Imon, S. Mikumo, and M. Katsuragi, "Tuning a bandpass filter by optical control of a negativeresistance circuit," IEEE Trans. Microwave Theory \& Tech. vol. 46, no. 12, pp. 2006-2010, Dec. 1998.

[12] J. Lee, Y.-T. Lee, and S. Nam, "A phase noise reduction technique in microwave oscillator using high $\mathrm{Q}$ active filter," IEEE Microwave and Wireless Components Lett., vol. 12 , no. 11, pp. 426-428, Nov. 2002

[13] M.-Q. Lee, S.-J. Yi, S. Nam, Y. Kwon, and K.-W. Yeom, "High efficiency harmonic loaded oscillator with low bias using a nonlinear design approach," IEEE Trans. Microwave Theory \& Tech., vol. 47, no. 9, pp. 1670-1679, Sept. 1999.

[14] J. Obregon and A. P. S. Khanna, "Exact derivation of the nonlinear negative-resistance oscillator pulling figure," IEEE Trans. Microwave Theory \& Tech., vol. 30, no. 7, pp. 1109-1111, July 1982.

[15] A. P. S. Khanna, E. Topacio, E. Gane, and D. Elad, "Low jitter silicon bipolar based VCOs for applications in High speed optical communication systems," 2001 IEEE MTT-S Int. Microwave Symp. Dig., vol. 3, pp. 1567-1570, May 2001. 